\title{
STRUCTURAL DIFFUSION, INTERFACE STRUCTURE AND CRYSTAL GROWTH
}

\author{
N. H. FLETCHER* \\ Department of Applied Physics, Faculty of Engineering, Hokkaido University, Sapporo, Japan
}

Received 10 June 1974; revised manuscript received 1 October 1974

\begin{abstract}
A description is developed of the structure of the interface between a crystal and its melt, and of the growth of a crystal in such a situation, which concentrates attention exclusively on the liquid side of the interface. It is concluded that structural effects extend to at least a distance of $20 \AA$ from the interface and that the interfacial free energy can be accounted for almost entirely by entropy loss in the liquid. A criterion is derived for surface roughness which has some relation to the similar criterion derived by Jackson. The theory is then applied to the growth of a crystal from its melt and an expression is derived for the minimum defect concentration in the crystal. It is concluded that the defects are largely vacancies or interstitial atoms and on this basis a calculation is made of the minimum dislocation density. This quantity is shown to increase linearly with growth velocity. Finally the theory is applied to consideration of the possible production of vitreous solids by very rapid freezing. As an illustrative example, calculations are given for the case of the water-ice interface.
\end{abstract}

\section{Introduction}

The growth of single crystals from pure melts appears now to be a subject which is well understood from the viewpoints of both physics and technology ${ }^{1,2}$ ). The structure of the liquid-solid interface is crucial in determining the details of the crystal growth mechanism and key contributions were made to the theory by the nucleation mechanism of $V_{\text {olmer }}^{3}$ ), the dislocation growth theory of Burton, Cabrera and Frank ${ }^{4}$ ) and most recently by the detailed considerations of interface structure initiated by the work of Jackson ${ }^{5}$ ).

It is notable, however, that all this work concentrates nearly exclusively on the structure of the crystal surface. The liquid is ignored, or simply considered as a passive partner whose role is to transport material to and heat away from the growing crystal. The few exceptions seem to be in the work of Turnbull ${ }^{6}$ ), Nason ${ }^{7}$ ) and Ewing ${ }^{8}$ ) on the structure of the equilibrium interface.

The object of the present paper is to take an entirely contrary approach and to concentrate attention on the liquid near the interface. The crystal will be regarded simply as a template specifying the structure of the solid phase but any other properties it may have will be largely ignored. We shall find that we can in this way construct a theory of crystal growth which is largely complementary to the usual theory, although its realm

* On leave from Department of Physics, University of New England, Armidale, N.S.W. 2351, Australia. of applicability is not so large and is certainly confined to crystal growth from the melt.

We must emphasize at the outset that the theory lacks rigour in some of its features and is frankly exploratory and speculative. The liquid viewpoint has been maintained exclusively to emphasize the difference from conventional theory but, if the points made are accepted as valid, it will be the task of the next generation of theories to combine the two approaches into a comprehensive description of the interface.

In this paper the theory will be presented in as general a manner as possible and then specific numerical application will be given for the case of the ice-water interface and the growth of ice crystals from water.

\section{Liquid structure near an interface}

What is usually called the structure of a liquid depends upon the time scale we have in mind, for the atoms or molecules in the liquid continually undergo vibrations with a time scale of order $10^{-13} \mathrm{~s}$ and diffusive rotations and translations with time scale of order $10^{-10} \mathrm{~s}$. The usual view, and the one to be adopted here, is that seen in a time of order $10^{-11} \mathrm{~s}$, so that vibratory motions are averaged out but diffusive motions cause slow changes in the structure with characteristic time $\tau \sim 10^{-10} \mathrm{~s}$.

If we consider the liquid structure averaged over a time long compared with $\tau$, then the only information available is the two-body correlation function $n_{2}\left(\boldsymbol{r}_{1} ; \boldsymbol{r}_{2}\right)$ 
which gives the probability that there will be an atom at $\boldsymbol{r}_{2}$, given that there is one at $\boldsymbol{r}_{1}$. This $\boldsymbol{n}_{2}\left(\boldsymbol{r}_{1} ; \boldsymbol{r}_{2}\right)$ is usually written in terms of the radial distribution function $g(r)$ as

$n_{2}\left(\boldsymbol{r}_{1} ; \boldsymbol{r}_{2}\right)=4 \pi r^{2} n_{\mathrm{L}} g(r)$.

where

$r=\left|r_{1}-r_{2}\right|$,

and $n_{\mathrm{L}}$ is the number density of atoms in the liquid. For a simple liquid like argon or sodium this description is adequate for many purposes and the superposition approximation can be used to obtain higher order correlation function like $n_{3}\left(\boldsymbol{r}_{1}, \boldsymbol{r}_{2} ; \boldsymbol{r}_{3}\right)$. For associated liquids like water, however, the superposition approximation is inadequate and a good deal of information about the structure of the liquid resides in the three-body correlation functions $n_{3}\left(\boldsymbol{r}_{1}, \boldsymbol{r}_{2} ; \boldsymbol{r}_{3}\right)$. For water, for example. this function specifies that nearest-neighbours are arranged nearly tetrahedrally with a bond angle which does not differ very greatly from the regular tetrahedral value.

From such information, then, we know something about the structure of the liquid. The radial distribution function $g(r)$, for example, always shows an exclusion volume near the origin, a nearest-neighbour peak and then successively less well defined peaks for more distant neighbours, as shown in fig. 1 . The typical range for the "structure" in $g(r)$ is about $10 \AA$.

When we consider liquid near a low-index crystal face of the same material, we can use the same principles to construct a distribution function for atoms in the liquid. Here however there is a two-dimensional array of atoms already fixed in position and each of these, on the liquid side, should participate in a radial distribution function similar to that for bulk liquid. This should certainly be true at least for the nearestneighbour shell, and the intersections of the probability distributions of nearest-neighbours for all the atoms in the crystal surface can be used to construct a probability distribution for the first liquid-like layer above the interface. In cases where the three-body correlation function is known approximately and this is different from that given by the superposition approximation, this information should also be used in the construction of the liquid probability distribution.

If we choose $y$ and $z$ axes in the interface and take $x$ normal to it, then this first-neighbour liquid layer will consist of an array of Gaussian probability ellipsoids which have, in the $1:$ a plane, the symmetry of the crystal surface. This is, of course, a statistical property and does not impose such symmetry on atomic arrangement in the layer.

Subsequent liquid-layers have their probability distributions determined in a similar way, building upon the ellipsoids of the first layer and adding squares of the standard deviations involved, as is appropriate for

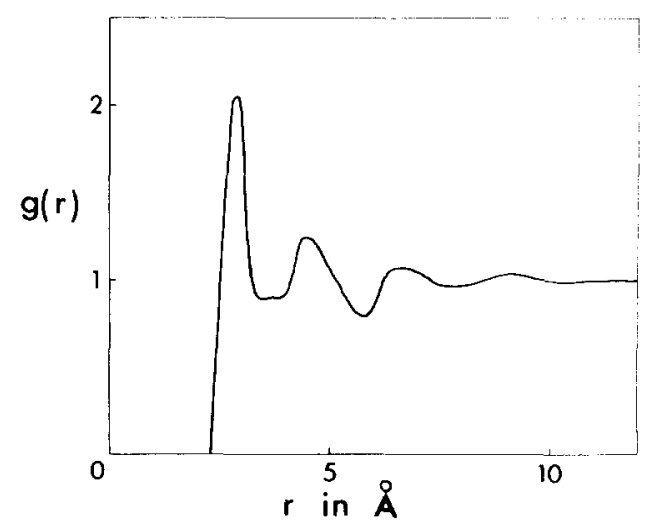

Fig. 1. A typical radial distribution function $g(r)$ for a liquid. This example is actually for water near 0 C but simple liquids have similar behaviour.

independent stochastic variables. Where the probability ellipsoids overlap, the individual probabilities are summed, so that the distribution eventually becomes uniform.

An example of the distribution function $P(x)$ is shown in fig. 2. There is an obvious layer structure in the liquid which extends for a distance of perhaps $20 \AA$ away from the interface. It is not surprising that this distance is rather longer than the characteristic distance in the radial distribution function. The decay of the bonding order is stochastic, and thus mathematically related to the diffusion equation. so that if the radial solution has the form

$\Sigma \sim r^{-1} \exp \left(-r_{i} l\right)$

where $\Sigma$ somehow measures order, then the one-dimensional case should behave like

$\Sigma \sim \exp (-x / /)$.

which effectively has a longer range.

A somewha1 similar discussion of liquid structure near an interface has been given by Ewing ${ }^{8}$ ) except that he has assumed, without discussion, that the distribu- 


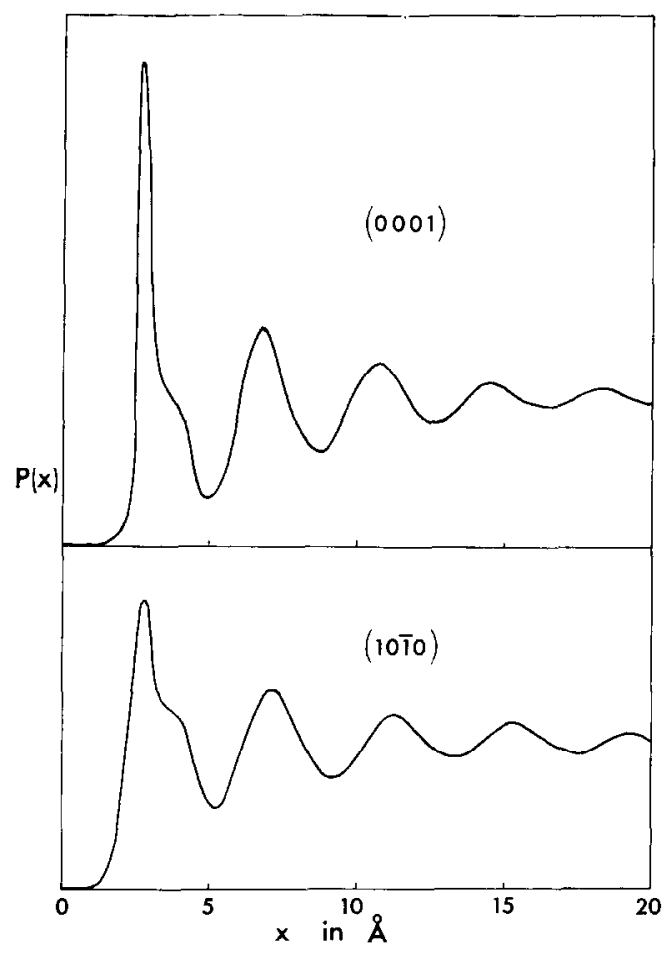

Fig. 2. Probability distribution $P(x)$ for atoms near a crystalliquid interface. The actual examples given are for water molecules near a (0001) or a (10̄̄0) face of ice but simple liquids have similar behaviour.

tion function normal to the interface, $P(x)$, is identical with the radial distribution function $g(r)$. There seems to be no justification for this assumption. The curves in fig. 2 are in fact calculated from the radial distribution function of fig. 1 for the case of the water-ice interface, using an explicit model for the liquid water structure, as we shall discuss later. The distinction between $P(x)$ and $g(r)$ is clear and there is no reason why a similar distinction should not exist for other liquids.

\section{Interfacial free energy}

For many types of interface it is customary to estimate the interfacial energy, for example by counting broken bonds, and then to make a small adjustment of some kind for entropy effects in order to obtain the free energy. For crystal-melt interfaces, however, as Turnbul1 ${ }^{5}$ ) has pointed out, the entropy contribution may be even more important than the energy term. This is born out by a calculation made for gold by Ewing ${ }^{8}$ ), though we have expressed above a reservation about some of his assumptions.

Ewing normalized the probability distribution $P(x)$ normal to the interface by dividing by the uniform liquid density $P_{0}$ as

$$
W(x)=P(x) / P_{0}
$$

and, integrating over a cylindrical volume with unit area in the interface, extending a distance $b$ into the liquid and containing $N$ atoms, showed that the contribution to the interface entropy is

$S=-N k \int_{0}^{1} W(X) \ln W(X) \mathrm{d} X$,

where

$X=x / b$.

Since $W=1$ in the bulk liquid, the result is independent of $b$. Similarly, since $W=0$ in the range $0<x<1.3 \AA$ as shown in fig. $2, S$ is also independent of the location of the plane taken to define the interface provided only that it lies within this region. The usual Gibbs dividing surface would be placed near $x=1.4 \AA$ so as to cause the surface excess mass to vanish, but this concept is not really applicable because of our artificial treatment of the crystal surface. We might also note that there may be some reservation about using the single-particle probability functions to calculate the entropy in this way, but we shall not pursue this question here.

In the more general case of an associated liquid where rotational freedom is also considered for each liquid molecule, (6) requires generalization. If, as seems a reasonable first approximation in some cases, the relative freedom of molecules in rotational phase space is proportional to their relative freedom in translational space, then the entropy will be twice the value given by (6). It is also really necessary to allow for the variation of $W$ in the $x y$ plane. With these possible extensions in mind we will continue to use (6) in its original form for the present.

Now once we have a knowledge of $P(x)$, and we shall discuss this for the case of water later, we can evaluate the surface entropy. Because the liquid near the interface is more ordered than is bulk liquid, $S$ is always negative. From his calculation for gold, Ewing found $S \simeq-0.3 k$ per surface atom, while we will see later that $S \simeq-k$ per atom for the ice-water interface and, in fact, we expect all interface entropies to be of this same order.

The other component of interfacial free energy arises 
from energy considerations. To first order, let us suppose that the liquid extends with bulk structure up to the interface and that within it the coordination number is $Z_{1}$, and the nearest-neighbour interaction energy $\varepsilon_{\mathrm{LL}}<0$. Similar quantities for the crystal are $Z_{\mathrm{S}}$ and $\varepsilon_{\mathrm{Ss}}$. Then by considering the energy changes when bulk liquid is separated along a plane, bulk crystal is separated along a plane, and liquid and crystal are joined to form an interface, we find that the energy associated with each molecule in the interface is

$E=\frac{1}{2} \beta\left[Z_{\mathrm{L} .}\left(\varepsilon_{\mathrm{LS}}-\varepsilon_{\mathrm{LL}}\right)+Z_{\mathrm{S}}\left(\varepsilon_{\mathrm{LS}}-\varepsilon_{\mathrm{SS}}\right)\right]$,

where $\varepsilon_{L S}$ is the nearest-neighbour interaction across the interface and $\beta$, which is approximately $\frac{1}{4}$, is the fraction of nearest neighbour links which extend across the interface. If we make the reasonable assumption that $\varepsilon_{\mathrm{LS}}=\frac{1}{2}\left(\varepsilon_{\mathrm{SS}}+\varepsilon_{\mathrm{LL}}\right)$, then $(8)$ can be written

$E={ }_{4}^{1} \beta\left(Z_{\mathrm{S}}-Z_{\mathrm{L}}\right)\left(\varepsilon_{\mathrm{LL}}-\varepsilon_{\mathrm{SS}}\right)$.

while the extreme assumption that $\varepsilon_{\mathrm{L} S \mathrm{~S}}=\varepsilon_{\mathrm{LL}}$ gives

$E=\frac{1}{2} \beta Z_{\mathrm{S}}\left(\varepsilon_{\mathrm{LL}}-\varepsilon_{\mathrm{SS}}\right)$.

Ewing makes the second assumption and uses the result (10) to derive an energy contribution for the gold interface which is very nearly equal to the contribution - TS from interface order. This probably represents an overestimate. For the water-ice interface, since $Z_{1} \simeq Z_{\mathrm{S}}$ due to the associated nature of the liquid, (9) predicts $E=0$ so that the entropy effect may provide essentially all the interfacial free energy.

Nason ${ }^{7}$ ) goes rather further than this and considers changes in interaction energy over the whole region in which the distribution is non-uniform. This is probably correct but is likely to represent a rather small correction.

The total interfacial free energy $\gamma$ is given by

$\gamma=N(E+T S)$,

where $N$ is the number of atoms per unit area of interface. Since $S$ is negative and $-T S>E$, we expect $\gamma$ to increase quite strongly with increasing temperature.

The water-ice interfacial free energy has been studied both near $-40^{\circ} \mathrm{C}$ by homogeneous nucleation experiments and near 0 C by a variety of methods and, as we shall discuss later, shows an increase from $22 \pm 3$ to $35 \pm 10 \mathrm{erg} \mathrm{cm}^{-2}$ over this range, in agreement with expectations.

We conclude, therefore, in support of our original thesis, that the equilibrium structure of the liquid near a crystal-melt interface plays an important, and perhaps even dominant part in determining the interfacial free energy.

\section{Interface structure}

In the conventional approach to crystal growth the notion of interface roughness plays a key part, one of the simplest theories being that of Jackson ${ }^{5}$ ). He considers a crystal surface for which the fraction of nearestneighbour bonds lying in the surface plane is $x$ and for which the latent heat of fusion is $L$ per atom at molting temperature $T_{\mathrm{m}}$. The theory then shows that there are two possible cases: either the number of crystal-like atoms in the surface layer is nearly 0 or nearly 1 , in which case the interface is "smooth", or else this number is close to 0.5 , in which case the interface is "rough". The criterion derived from the theory is that the interface will be rough if

$\mathrm{L} / 2 k T_{\mathrm{m}}<1 / x$.

In the present theory the concept of surface roughness plays no direct part but it is possible to derive a nearly equivalent description of the surface and a criterion related to (12). In our interface model, layer zero is unambiguously crystalline but the first layer, though considered as liquid, has an entropy lower than the bulk liquid. This entropy is a statistical quantity, and, if we were to adopt a "mixture" model for the liquid structure, could be interpreted as a superposition of molecular configurations with crystal-like arrangement and others with liquid-like arrangement. The criterion that the interface be "rough" in the previous sense is therefore that this first layer have an entropy $S(1)$ which is less than half the entropy of the liquid $S_{0}$, the crystal having zero configurational entropy. Since we have used the liquid entropy $S_{0}$ as a calculation base for $S$, this condition can be written

$S_{0}-S(1)>\frac{1}{2} S_{0}$.

Our model is actually a multi-layer one, in contrast with Jackson's single-layer model, so that if $S(1) \ll S_{0}$, in fulfilling (13) we will have a very diffuse rough interface extending over several molecular layers.

In discussing the structure of this first liquid layer it is not necessary, physically, to introduce the idea of bonds, except in the case of associated liquids. Since, however, each crystal atom at the interface serves as a 
centre about which the nearest-neighbour distribution is constructed using superposed radial distribution functions, it is convenient to define a "bond" for our present purpose as simply a vector joining nearestneighbour atoms across the interface.

To evaluate $S(1)$ approximately, we note that the nearest-neighbour maximum in a typical radial distribution function is approximately Gaussian so that the intersection of two or more radial distribution functions leads to an approximately ellipsoidal probability distribution for any one atom, which can reasonably be approximated by a 3-dimensional Gaussian distribution with standard deviations $\sigma_{1}, \sigma_{2}, \sigma_{3}$. However if the coordination number in the crystal is $Z$ and a fraction $\alpha$ of the bonds lie in the plane of the crystal surface, then each atom in layer 1 is constrained by $Z(1-\alpha) / 2$ "bonds", in the sense that it belongs to the first-neighbour correlation shell of this number of atoms in the crystal surface. Now the variances $\sigma_{i}^{2}$ are approximately inversely proportional to the number of constraints, so that the standard deviations $\sigma_{i}$ can reasonably be written

$\sigma_{i}=\mu_{i}[Z(1-\alpha) / 2]^{-1 / 2}$,

where the $\mu_{i}$ are characteristic of the interatomic potential and the geometry of the surface.

We may now use (6) with $N=1$ and $b=v_{0}^{1 / 3}$, where $v_{0}$ is the volume per atom in the liquid, to estimate the difference between the entropy of layer 1 and the bulk liquid. The result is

$S_{0}-S(1) \simeq k \sum_{i=1}^{3} \ln \left\{v_{0}^{1 / 3} \mu_{i}^{-1}[(1-\alpha) Z / 4 \pi \mathrm{e}]^{1 / 2}\right\}$,

but, because of the approximations made, we must require that each $i$ term in (15) be either positive or zero. A more detailed consideration would obviate this difficulty but is not necessary for our present purpose.

The values of the quantities $\mu_{i}, v_{0}, Z$ and $\alpha$ appearing in (15) are known, at least in principle, from the radial distribution function and the geometry of the crystal surface so that we can write

$S_{0}-S(1)=k F(\mathrm{RDF}, \alpha)$

where $F$ is an undetermined function of order of magnitude unity. Returning to (13), the right side can be simply written as $L / 2 T_{\mathrm{m}}$, where $L$ is the latent heat of fusion per atom and $T_{\mathrm{m}}$ the melting temperature, so that the criterion that the interface be rough becomes

$L / 2 k T_{\mathrm{m}}<F(\mathrm{RDF}, \alpha)$.

The two criteria (12) and (17) are clearly not identical, and even depend on different quantities. The right side of (17) is, however, of order of magnitude unity and decreases as $\alpha$ increases so that the two results show the same general behaviour. We shall see that, for the water-ice interface, the criteria (12) and (17) are essentially equivalent.

One other aspect of interface structure merits brief mention here and that is the step. A surface with a step can, in fact, be simply defined in our model since it only involves a step in the crystal template. Liquid atoms near the step will be bonded to crystal positions in the step as well as in the face itself so that their configurational entropy will be further reduced, implying an edge free energy associated with the step. Because, however, the step entropy can decay diffusively in two dimensions in the liquid rather than just in one, it is likely that for steps only a few atomic layers high the free energy will be less than that associated with an equal area of plane surface.

\section{Structural diffusion and crystal growth}

We now come to apply these ideas to the process of crystal growth. In keeping with our philosophy in this paper we shall ignore the problem of accommodation of atoms in the growing surface and concentrate exclusively on the structural rearrangements in the liquid near the interface. Any rigorous discussion of these rearrangements should consider the unit stochastic processes of atomic translations and, in the case of a molecular liquid, of rotations as well. Such a description is necessarily complicated and we shall see that we can, in fact, go a considerable way with the theory by taking a more coarse-grained view and treating the problem as one of structural diffusion in an ordering field provided by the crystal surface. As with all diffusion problems, we may expect this approximation to be valid provided that the characteristic length associated with the diffusion gradients is sufficiently much greater than the characteristic length associated with the elementary stochastic processes (i.e. the "mean free path"). In the present case the length $\delta$ associated with the elementary processes is about one atomic or molecular radius, say $2 \AA$, while the characteristic structural diffusion length 
$l$ is about $10 \AA$. The diffusion approximation is therefore reasonable, though we should not expect great quantitative accuracy from its predictions.

In an equilibrium situation there is a gradient of order, and hence of configurational entropy, in the liquid near the interface. This entropy gradient is measured qualitatively by the amplitude of the density oscillations in the structure function near the interface, as shown in fig. 2, and we must now make this concept more quantitative.

Referring to eq. (6), we defincd there an cntropy function $S$ giving the entropy difference between $N$ molecules in a cylinder of height $X=1$ and base lying at the interface, and $N$ molecules of bulk liquid. If instead of taking this integral over the range $X=0$ to 1 we take only the range $X$ to $X+A$, then the new quantity $\bar{Q}$ defined by the integral is the local deviation from bulk liquid entropy at distance $x=b X$ from the interface. For this prescription to be fully satisfactory we require that $\delta \ll \Delta \ll /$ and we have seen that it is only marginally possible to satisfy this condition for the interface problem. Nevertheless we continue the development in a heuristic spirit.

This whole approximation breaks down, however, in the case where the interface is really smooth in terms of Jackson's criterion for then, as we discussed earlier, the configurational entropy of the first liquid layer is closer to that of bulk liquid than to that of solid. In this case the characteristic length $l$ is less than the elementary length $\delta$ so that the whole following theory must be recast in terms of elementary stochastic processes. This we do not attempt here to do. The theory which follows therefore applies only to situations where the interface is rough, or nearly rough, in terms of our criterion (17) or the similar criterion of Jackson.

A qualitative appraisal of fig. 2 indicates that the local entropy function $\Sigma=\bar{Q}+S_{0}$ rises from zero in the crystal to a value $\Sigma_{0}=S_{0}$, characteristic of the bulk liquid, in a quasi-exponential manner, as shown in fig. 3. We shall examine the construction of this function in more detail when we come to consider the water-ice interface but for the moment we suppose it to have the form

$\Sigma=\Sigma_{0}[1-\exp (-x / /)]$.

Consider now an atomic layer or group of layers at a distance $x$ from the interface. Their equilibrium local

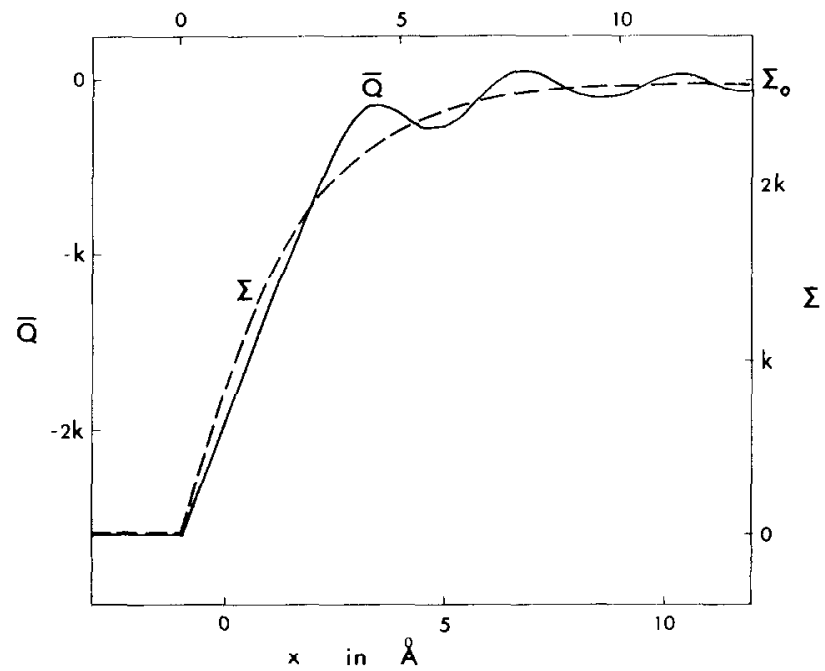

Fig. 3. Behaviour of the local entropy function $\Sigma$ in the liquid near an interface. The quantity $Q$ has been evaluated for an integration interval $A-2 \AA$ for the case of water near an (0001) ice plane and then further smoothed to give $\Sigma$. The lower a scale is relative to the geometrical ice surface while in the upper scale (used in the calculations) the origin has been displaced to coincide with the point where $\Sigma-0$.

entropy $\Sigma$ is given by (18) and is maintained in a dynamic way by processes of bond rearrangement or molecular diffusion, these processes having a characteristic time scale $\tau$ which is known, from neutron scattering experiments, to be of order $10^{-10} \mathrm{~s}$ for most liquids. Suppose now that this equilibrium $\Sigma$ is upset in some way to a new value $\Sigma^{\prime}$. We should then expect, on removal of the disturbing influence, that $\Sigma^{\prime}$ would decay back towards its local equilibrium value according to the equation

$\partial \Sigma^{\prime} / \hat{\imath} t=-\left(\Sigma^{\prime}-\Sigma\right) / \tau$.

Now let us consider not the static situation but rather the case in which the crystal is growing with velocity $v$ in the $x$-direction. If we choose a coordinate system moving with the interface, then the differential equation for $\Sigma^{\prime}$, the instantaneous value of $\Sigma$ at a distance $x$ from the interface, becomes

$\frac{\partial \Sigma^{\prime}}{\partial t}=-\frac{\Sigma^{\prime}-\Sigma}{\tau}-v^{2} \partial \Sigma^{\prime}$.

or, using (18) and omitting the prime from $\Sigma^{\prime}$,

$\tau \frac{\partial \Sigma}{\partial t}=-\Sigma+\Sigma_{0}\left(1-\mathrm{e}^{-x / t}\right)+v \tau \frac{\partial \Sigma}{\partial x}$.

Now what we are interested in is the steady state solu- 
tion of this equation, $\partial \Sigma / \partial t=0$, corresponding to a steadily advancing interface and satisfying $\Sigma=\Sigma_{0}$ at $x=\infty$. This is

$\Sigma=\Sigma_{0}-\Sigma_{0} \mathrm{e}^{-x / l} /(1+v \tau / l)$.

For positive $v$ the partially ordered region is therefore restricted to a slightly closer distance from the interface than in its equilibrium state.

The thing which now interests us is the boundary condition at $x=0$, which is

$\Sigma(0) \equiv \Sigma_{1}=\frac{\Sigma_{0} v \tau / l}{1+v \tau / l}$.

Clearly there cannot be a discontinuity in $\Sigma(x)$ at any point under our continuous diffusion approximation, so that we conclude that the configurational entropy of the growing crystal is no longer zero but rather $\Sigma_{1}$. In ordinary crystal growth $v<1 \mathrm{~cm} \mathrm{~s}^{-1}$ and we have already seen that $\tau \sim 10^{-10} \mathrm{~s}$ and $l \sim 10^{-7} \mathrm{~cm}$, so that (23) suggests

$\Sigma_{1} \sim 10^{-3} v \Sigma_{0}$,

when $v$ is in $\mathrm{cm} \mathrm{s}^{-1}$.

This result tells us nothing about growth kinetics, but it does place a lower limit on the defect concentration which can be achieved in growth from the melt, and predicts that this concentration should vary linearly with growth speed. We do not yet have any information about the kinds of imperfections contributing to $\Sigma_{1}$, but it is possible to make some statements about these.

In a liquid the configurational disorder may be of many kinds but all may be described geometrically as bonding disorders. When this sort of disorder is built into the growing crystal it must be largely as point defects, although of course any disorder which looks like a dislocation line normal to the interface will continue into succeeding crystal layers. The only point defects describable as bond disorders in a pure crystal are vacancies and interstitial atoms and we may therefore expect $\Sigma_{1}$ to be derived primarily from these defects.

Now in general the density of a liquid differs from that of its parent solid by 5 to $10 \%$, the liquid being less dense than the crystal for closely packed crystals and more dense for open crystals having, for example, tetrahedral coordination. In the first case, therefore, we expect the prime defects incorporated in the crystal to be vacancies, while in the second case interstitial atoms should predominate. If the mole fraction of such point defects, assumed all one kind, in the crystal is $c$, then the entropy associated with them is, for small $c$,

$\Sigma_{1} \simeq k c \ln c$.

Substitution in (24) and approximatc solution in the range $10^{-10}<c<10^{-1}$ gives

$c \sim 10^{-4} v \Sigma_{0} / k$.

For most simple materials, however, $\Sigma_{0}$ can be equated to the entropy of fusion which is of order $k$, so that we can write

$c \sim 10^{-4} v$,

or, taking the number of atoms per unit volume in the crystal as $10^{23} \mathrm{~cm}^{-3}$, we find a total defect density $n$ of $n \sim 10^{19} v$,

where $n$ and $v$ are in c.g.s. units.

If these vacancies or interstitials are now regarded as congregating into discs of radius $r$ which then coalesce to form edge dislocations, then a simple calculation gives the dislocation density as

$N \sim 10^{-15} n / r$.

The final result clearly depends critically upon the choice of $r$, but $r \sim 10^{-2} \mathrm{~cm}$ may be typical. Combination of (28) and (29) then gives

$N \sim 10^{6} v$,

so that, for a typical growth rate of $10^{-4} \mathrm{~cm} \mathrm{~s}^{-1}$ we might expect a minimum dislocation density of order $10^{2} \mathrm{~cm}^{-2}$. This is uncertain by at least one order of magnitude because of uncertainty in $r$. It does, however, appcar to be an estimate in order-of-magnitude agreement with experimental experience.

Finally it is interesting to calculate the crystal growth rate necessary in order to include almost all the liquid disorder $\Sigma_{0}$ in the solid, thus producing a vitreous structure. Consideration of eq. (23) shows that this is not strictly possible unless $v \rightarrow \infty$, but that $\Sigma_{1}=\frac{1}{2} \Sigma_{0}$, so that half of the disorder is included, if

$v=v^{*}=l / \tau$.

From the numerical values discussed previously, this implies

$v^{*} \sim 10^{3} \mathrm{~cm} \mathrm{~s}^{-1}$. 
The critical crystallization velocity predicted by $(32)$ is, of course, very high, but may not be beyond the realm of possibility for a splat-cooling process in which the liquid droplet is made to splash to a thin film on a block of metal cooled to liquid nitrogen temperature.

\section{Discussion}

The theory which we have presented is quite general and should apply to virtually all crystal-melt systems. As pointed out in the introduction, the liquid side of the interface has been considered almost exclusively and no attempt has been made to produce the unified treatment of both crystal and liquid sides of the interface which must almost certainly appear as the final theory.

Our discussion has given what appear to be fairly satisfactory descriptions of interface geometry and free energy, and the development has also allowed a prediction of the minimum defect content of a growing crystal, a quantity which has not emerged from other treatments.

As far as growth kinetics are concerned the theory is silent, but so indeed is a theory based solely upon the crystal side of the interface. The usual approach is to use the crystalline description of the interface simply to provide a measure of the number of accommodation sites and then to use a diffusion calculation of equilibrium flux rates to derive growth velocity.

It should be possible to apply a somewhat similar approach to the present case. There is no consideration of the number of accommodation sites in the crystal, since all are assumed available, but the diffusion processes near the interface are across a multiple set of energy maxima whose properties differ with distance from the interface. We should expect a kinetic result which is always linear in the supercooling $\Delta T=\left(T_{\mathrm{m}}-T\right)$ but whose kinetic coefficient depends upon liquid structure near the interface.

With these qualifications, then, the theory above is presented as one possible extreme view of the interface between a crystal and its melt. It is hoped that by presenting this extreme view a synthesis can be provoked with existing theory.

\section{The water-ice interface}

In the following sections of this paper we apply the general theory developed above to a specific case so that more detailed estimates of the various quantities involved can be made for comparison with experiment. The system chosen for this application is the water-ice interface, not because it is a simple system - it is not but because it is a system of considerable fundamental and applied interest for which a reasonable amount of experimental information is available.

Water is an associated liquid with a coordination number near 4 . Its structure thus depends importantly on the three-body correlation function which specifies average bond angles and their fluctuations. A recent review of theorics of liquid water has been given elsewhere ${ }^{10}$ ), from which it is clear that there are two competitive classes of models - the uniform model of Pople ${ }^{11}$ ) in which distorted tetrahedral bonding is maintained almost everywhere, and the various mixture models which treat water as composed of clusters of molecules with well-defined but differing bonding patterns. Both these models are extreme idealizations and the truth lies somewhere in between. The two approaches agree, however, in assigning a coherence length for bonding ${ }^{10}$ ) in liquid water of about 5-10 $\AA$. It may well be that five-fold rings play a major part in water structure ${ }^{12-14}$ ) but this need not concern us specifically here.

For our present purpose, the uniform, distorted-bond model of Pople ${ }^{11}$ ) is most convenient for calculation. From comparison with the radial distribution function near $0{ }^{\circ} \mathrm{C}$ he deduced that bond lengths are normally distributed about a mean of $2.8 \AA$ with standard deviation $\delta r=0.26 \AA$, while the standard deviation of bond angles from their tetrahedral value is $\delta \phi=26^{\circ}$. We shall adopt these values for our model.

\section{Interface structure and free energy}

Following the method set out above we can now construct the probability distribution function for liquid water near an ice surface. The calculated distributions normal to the surface are shown in fig. 2 for the case of an $(0001)$ and a (10T0) interface. It is clear from these curves that structural effects extend away from the interface for a distance of at least $20 \AA$.

When we come to calculate the entropy deficit and hence the free energy of the interface according to eq. (6), it is clear that a 3-dimensional integration is really involved, not just one over the distributions shown in fig. 2. Carrying out this computation as outlined in section 4 , we find for the surface entropy deficits 
$S_{(0001)} \simeq-1.0 k, \quad S_{(10 \overline{\mathrm{T}} 0)} \simeq-0.8 k$,

which, at $0^{\circ} \mathrm{C}$, correspond to interface free energies

$\gamma_{(0001)} \simeq 40 \mathrm{erg} \mathrm{cm}^{-2}, \quad \gamma_{(10 \mathrm{TO})} \simeq 30 \mathrm{erg} \mathrm{cm}^{-2}$.

with both these values being proportional to absolute temperature.

Before accepting these as the values to be compared with experiments, we must consider two points. The first concerns the energy contribution to $\sigma$. It seems reasonable for water, and indeed for most materials, to adopt the approximation that the energy of the bonds across the interface is close to the mean of the solid and liquid interaction energies. Since the coordination number for water is almost the same as for ice, (9) then suggests that the energy contribution to $\sigma$ is small.

The second point concerns the rotational entropy loss associated with the interface region. This has certainly not been considered and our discussion has suggested that it may be comparable with the spatial entropy loss, a conclusion which would double the $\sigma$ values given above. On the other hand the orientational entropy of ice allows considerable orientational freedom through proton rearrangement and this is shared by molecules in the transition layer, reducing this contribution to the entropy by a large factor so that it can reasonably be neglected.

Experimental values of the average ice-water interfacial free energy have been determined in various ways ${ }^{10.15}$ ). At $-40^{\circ} \mathrm{C}$ the probable value is in the range $18-25 \mathrm{erg} \mathrm{cm}^{-2}$, while at $0{ }^{\circ} \mathrm{C}$ the range is $25-40$ $\mathrm{erg} \mathrm{cm}^{-2}$. The theoretical values are thus in acceptable agreement with experiment considering some of the uncertainties involved. $\gamma_{10001}$ is strongly dependent on the bond length fluctuation $\delta r / r_{0}$ so that the difference between $\gamma_{(0001)}$ and $\gamma_{(10 \mathrm{~T} 0)}$ may not be significant.

It is perhaps worth note in passing that crystal growth experiments by Hillig ${ }^{16}$ ) involving a surface nucleation process on (0001) surfaces suggested an edge free energy only about one third of that which would be expected from the step height and the plane surface free energy. This is in agreement with the conclusions reached from the theory, though there may be other explanations.

When we come to consider the roughness or smoothness of the interface according to Jackson's model ${ }^{5}$ ) we find $\alpha=0.75$ so that, in (12),

$L / 2 k T_{\mathrm{m}}=1.33=1 / \alpha$, and the interface is just on the border between rough and smooth behaviour. If we follow the prescription of eq. (15) we find for a (0001) interface that two of the terms in the summation have the negative value -0.18 $k$ while the third is $+1.4 k$. Setting the two negative terms equal to zero we find, in (16), $F(\mathrm{RDF}, \alpha)=1.4$ and again this is approximately equal to the left side of (35) so that the interface cannot be characterized clearly. For a (1010) interface, all three terms are positive and $F(\mathrm{RDF}, \alpha)=1.2$ which is again not significantly different from the left side of (35). The new theory thus reaches essentially the same conclusion about sharpness of the interface as does Jackson.

\section{Crystal growth and perfection}

To apply the theory of crystal growth and perfection developed above, we must first evaluate the finegrained entropy function $\bar{Q}$ defined in section 5 . This function is plotted, for a (0001) interface after averaging over a $2 \AA$ interval $\Delta$, in fig. 3 . The small oscillations in $\bar{Q}$ show that $\Delta$ should really be larger than this but the rather sharp variation of $\bar{Q}$ with $x$ restricts this. Further smoothing gives the function $\Sigma$, which is also shown in fig. 3. The important parameter which emerges is the structural diffusion length $/$ associated with $\Sigma$, which we estimate as $l=3 \times 10^{-8} \mathrm{~cm}$. This length is closely comparable with the bond length and hence with the elementary stochastic length for the problem, so that diffusion theory is only marginally applicable, as indeed we would expect from the indeterminacy of the surface roughness. Despite this, we proceed to evaluate the other predictions of the theory in the belief that they should be at least of the correct order of magnitude.

The characteristic time $\tau$ should be the relaxation time associated with the Debye dispersion in the dielectric constant of liquid water ${ }^{17}$ ). This has been found to occur at an electromagnetic wavelength near $3 \mathrm{~cm}$ at $0{ }^{\circ} \mathrm{C}$, which implies $\omega=6 \times 10^{10} \mathrm{~s}^{-1}$ and so $\tau \simeq 1.7 \times$ $10^{-11} \mathrm{~s}$. The other quantity required is $\Sigma_{0}$, which we identify with the entropy of fusion per molecule and write as $\Sigma_{0}=2.7 k$ so that eq. (24), for the entropy of disorder built into a crystal growing with velocity $v$, becomes $\Sigma_{1} \simeq 8 \times 10^{-4} v k$.

Now water is about $8 \%$ more dense than ice so that we expect most of this entropy to be built in as interstitial molecules. For $v$ in the range $10^{-5}$ to $10^{-3} \mathrm{~cm} \mathrm{~s}^{-1},(25)$ 
gives a total interstitial density of $n \sim 3 \times 10^{18} v$. Assuming that these aggregate to discs of radius $r \sim 10^{-2}$ $\mathrm{cm}$ and that these discs then combine to give edge dislocations implies a dislocation density $N$ of order

$N \sim 10^{5} v$,

or about $10 \mathrm{~cm}^{-2}$ for a growth velocity of $10^{-4} \mathrm{~cm} \mathrm{~s}^{-1}$. This should be interpreted as the lowest obtainable dislocation density, since dislocations can be introduced during growth from a variety of other sources.

Higashi ${ }^{18}$ ) has reported the growth of ice crystals of high perfection by several methods. The Czochralski method gave dislocation densities about 3 orders of magnitude larger than predicted by (36), though the dislocation density was proportional to growth rate. These dislocations are presumably nearly all extrinsic. A modified Bridgman method achieved $N \sim 10^{2} \mathrm{~cm}^{-2}$ for $v \sim 10^{-5} \mathrm{~cm} \mathrm{~s}^{-1}$, which is about two orders of magnitude larger than given by (36). The predictions of the theory are thus reasonable in terms of giving a minimum dislocation content, although they may be low by an order of magnitude.

Finally we examine the possibility of producing a vitreous ice structure by rapid growth as in splat cooling. From eq. (31) the critical velocity is $v^{*}=1 / \tau \sim$ $2 \times 10^{3} \mathrm{~cm} \mathrm{~s}^{-1}$. Now the average growth rate when a film of thickness $d$ is cooled on a block of infinite conductivity held at temperature $T_{\mathrm{b}}$ is approximately

$v \simeq 2 K\left(T_{\mathrm{m}}-T_{\mathrm{b}}\right) / L_{\mathrm{v}} d$,

where $L_{v}$ is the latent heat per unit volume and $K$ is the thermal conductivity of ice. Inserting appropriate values in (37) for $T_{\mathrm{b}}=77 \mathrm{~K}$, we find for the critical film thickness $d \simeq 1 \times 10^{-4} \mathrm{~cm}$. It seems impracticable to try to produce a splat film as thin as this with ordinary techniques so that this method of producing vitreous ice is probably not feasible.

\section{Conclusions}

The semi-quantitative application of our theory to the water-ice interface demonstrates that its predictions are generally in accord with experience. This does not, of course, guarantee the correctness of the assumptions but does give an increased measure of confidence.

The theory as set out here is only the first exploratory step towards a development with satisfactory rigor.
The rather small ratio between the elementary stochastic length and the structural diffusion length makes a microscopic theory almost mandatory in such a development. A proper working out of these mechanisms at the atomic level together with proper inclusion of effects on the crystal side of the interface together constitute the next part of the programme.

\section{Acknowledgements}

This work was carried out while the author was a Visiting Professor in the Faculty of Engineering at Hokkaido University under the auspices of the Japan Society for the Promotion of Science. He is most grateful to Professor Akira Higashi for hospitality in his laboratory during tenure of the position and for valuable discussions during the course of the study. The continuing programme is supported by the Australian Research Grants Committee.

\section{References}

1) R. L. Parker, in: Solid State Physics, Vol. 25, Eds. H. Ehrenreich, F. Seitz and D. Turnbull (Academic Press, New York, 1970) pp. 151-299.

2) R. A. Laudise, The Growth of Single Crystals (Prentice Hall, Englewood Cliffs, N.J., 1970).

3) M. Volmer, Kinetik der Phasenbildung (Steinkopf, Dresden, 1939).

4) W. K. Burton, N. Cabrera and F. C. Frank, Phil. Trans. Roy. Soc. London A 243 (1951) 299.

5) K. A. Jackson, in: Crystal Growth, Ed. H. S. Peiser (Pergamon, Oxford, 1967) pp. 17-24.

6) D. Turnbull, in: Physics of Non-Crystalline Solids, Proc. Intern. Conf. Delft, Ed. J. A. Prins (North-Holland Amsterdam, 1965) p. 41 .

7) D. O. Nason. The Structure of the Solid-Liquid Interface. Ph.D. Thesis, Stanford University (1970).

8) R. H. Ewing, J. Crystal Growth 11 (1971) 221.

9) N. H. Fletcher, The Chemical Physics of Ice (Cambridge University Press, Cambridge, 1970) pp. 85-97.

10) N. H. Fletcher, Rept. Progr. Phys. 34 (1971) 913.

11) J. A. Pople, Proc, Roy. Soc. (London) A 205 (1951) 163

12) F. C. Frank, Proc. Roy. Soc. (London) A 215 (1952, 43

13) J. D. Bernal, in: Liquids: Structure, Properties, Solid Interactions, Ed. T. J. Hughel (Elsevier, Amsterdam, 1965) pp. $25-50$.

14) L. Pauling, in: Hydrogen Bonding, Ed. D. Hadzi (Pergamon, Oxford, 1959) pp. 1-5.

15) D. R. H. Jones and G. A. Chadwick. J. Crystal Growth 11 (1971) 260 .

16) W. Hillig, in: Growth and Perfection of Crystals, Eds. R. H. Dorcmus, S. W. Roberts and D. Turnbull (Wiley, New York. 1958) pp. 350-360.

17) D. Eisenberg and W. Kauzmann, The Structure and Properries of Water (Clarendon Press, Oxford, 1969) pp. 205-222.

18) A. Higashi, J. Crystal Growth 24/25 (1974) 102. 